\title{
Tool Path Generation for the Formation of a Geometric Surface Pattern on a Curved Surface by Patch Division Milling*
}

\author{
Hiroshi MATSUDA $^{* *}$, Hiroyuki SASAHARA ${ }^{* * *}$, Masaaki FUKUDA ${ }^{* * *}$ \\ and Masaomi TSUTSUMI ${ }^{* * *}$ \\ **Department of Monodukuri Engineering, Tokyo Metropolitan College of Industrial Technology, \\ 8-17-1, Minamisenjyu, Arakawaku, Tokyo, 116-0003, JAPAN \\ E-mail: matsuda@acp.metro-cit.ac.jp \\ ***Bio-Applications and Systems Engineering, Tokyo University of Agriculture and Technology, \\ 2-24-16, Naka-cho, Koganei-shi, Tokyo, 184-8588, JAPAN
}

\begin{abstract}
A textural surface pattern is used to add a physical or decorative function to an object's surface. We have proposed a milling technique involving patch division that can quickly generate a geometric surface pattern using a common profile-forming method. This report describes a method of generating a tool path using 3D-CAD and an FEA preprocessor applied to the patch division milling of a curved surface. Experimental results of the proposed method as applied to spherical and free-form surfaces are described.
\end{abstract}

Key words: Surface Pattern, Cutter Marks, Ball-End Mill, Tool Path, Machining Center

\section{Introduction}

A surface pattern is often applied to a variety of products. A surface pattern can add a specific physical function to an object's surface, such as allowing the control of wettability or a water repellent quality ${ }^{(1)-(3)}$, or decreasing of the drag forces ${ }^{(4)}$ and abrasion between sliding parts ${ }^{(5)}$. Additionally, a surface pattern can be configured as an ornamental design on the exterior of such items as wall tiles, figured glass, etc. Therefore, the ability to form such surface patterns on a curved surface would be advantageous for the machinery industry.

A surface pattern can be generated chemically, or by machining methods such as cutting, grinding, or shot-blasting. In the present study, we have focused on one of the most generally used profile-forming methods which is relatively inexpensive and involves a short machining time. The end mill is often used for the generation of surface patterns. In particular, the ball-end mill is widely used to machine complex-shaped workpieces with a machining center.

Various surface patterns can be generated on the machined surface of a workpiece by means of ball-end milling ${ }^{(6)}$, allowing the realization of a surface pattern with a constant scallop height and a regularly aligned cutter mark array. Research has been carried out regarding the mechanism of generating a surface pattern by ball-end milling. For example, $\mathrm{X}$. Zhao et al. ${ }^{(7)}$ showed the relationship between cutting conditions and surface roughness. M. Hao et al. ${ }^{(8)}$ theoretically analyzed ball-end milling to elucidate the generating mechanism of machined surfaces, while Jenq-Shyong Chen et al. ${ }^{(9)}$ described the relationship between scallop height, tool radius, tool-axis inclination angle, and feed/pick ratio. A. Saito et al. ${ }^{(10)(11)}$ demonstrated that an optional surface pattern can be generated by adjusting the eccentricity and the traverse distance along the tool path. However, in all of these studies, the workpiece is assumed to have a limited shape. When the surface of a complex-shaped workpiece is machined, creases can be caused by rapid changes in the tool 


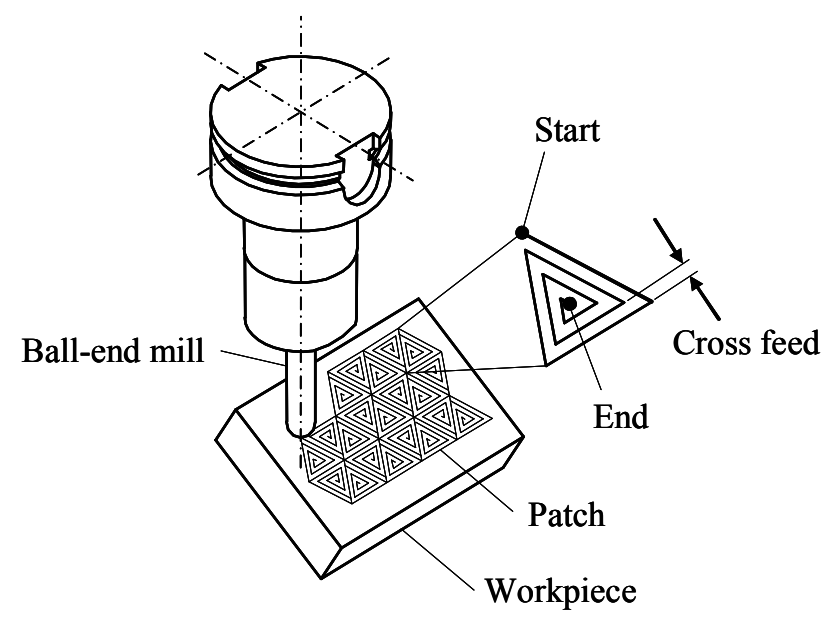

(a) Division of the machined surface and the tool path in a patch

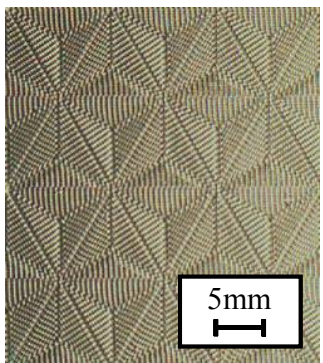

Overall view

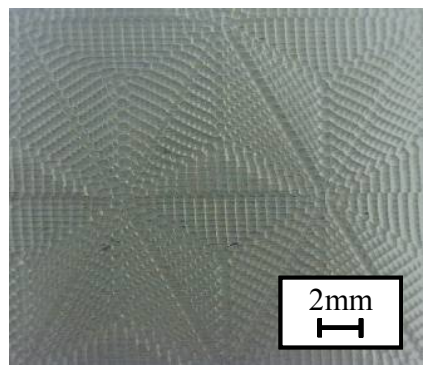

Enlarged view

(b) Machined inclined flat surface

Fig. 1 Outline of patch division milling

direction and in the tool-tilting angle; as a result, the surface roughness and form accuracy deteriorate markedly. Therefore, it is very difficult to generate a regular surface pattern in a workpiece having a complicated shape.

To resolve this issue, we have proposed a patch division milling technique that can promptly generate a geometric surface pattern through the use of ball-end milling ${ }^{(12)(13)}$. Patch division milling is developed to form regularly aligned surface patterns both by the cutter marks and the patch outlines. The surface is divided into a lot of just about same-size patch segments. The entire inside area of each patch is machined along a helical tool path. A surface pattern is generated by the cutting edges of the ball-end mill within each patch area.

The purpose of this study is to extend the application of patch division milling to a curved surface. In this paper, patch division milling and a method of generating a tool path on an inclined flat surface and a curved surface are described. A curved surface designed by 3D-CAD is divided into many triangular patches by an FEA preprocessor, and a helical tool path is automatically generated on the entire area of each patch. Additionally, this tool path generation system is applied to a sphere and a free-form surface, and the experimental results are described.

\section{Outline of patch division milling and tool path generation for an inclined} flat surface

In this section, patch division milling and the generation of a tool path on an inclined flat workpiece surface are described. First, as shown in Fig. 1(a), the entire surface is divided into many small patches. Then, the entire inside area of each patch is machined along a helical tool path. After these procedures, as shown in Fig. 1(b), an aligned surface pattern is generated within a patch area by cutting with the ball-end mill. In addition, the entire surface can be covered with continuous aligned patches.

The helical tool path within a regular triangle patch can be calculated as described below. A regular triangle $\mathrm{A}_{0} \mathrm{~B}_{0} \mathrm{C}_{0}$ with side length $L$ is defined on the $\mathrm{X}_{\mathrm{p}} \mathrm{Y}_{\mathrm{p}}$ plane as shown in Fig. 


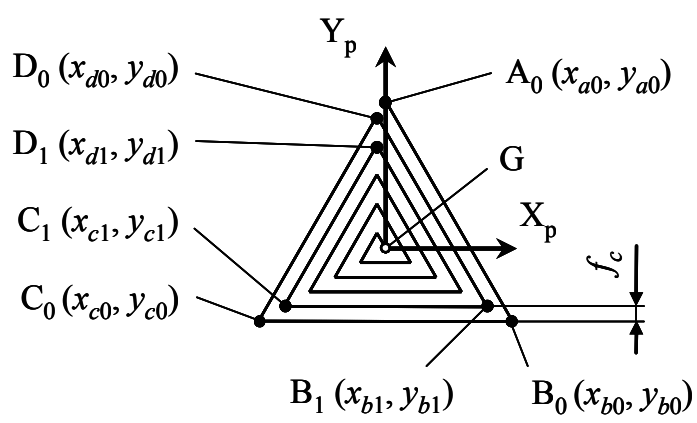

(a) Regular triangular reference patch

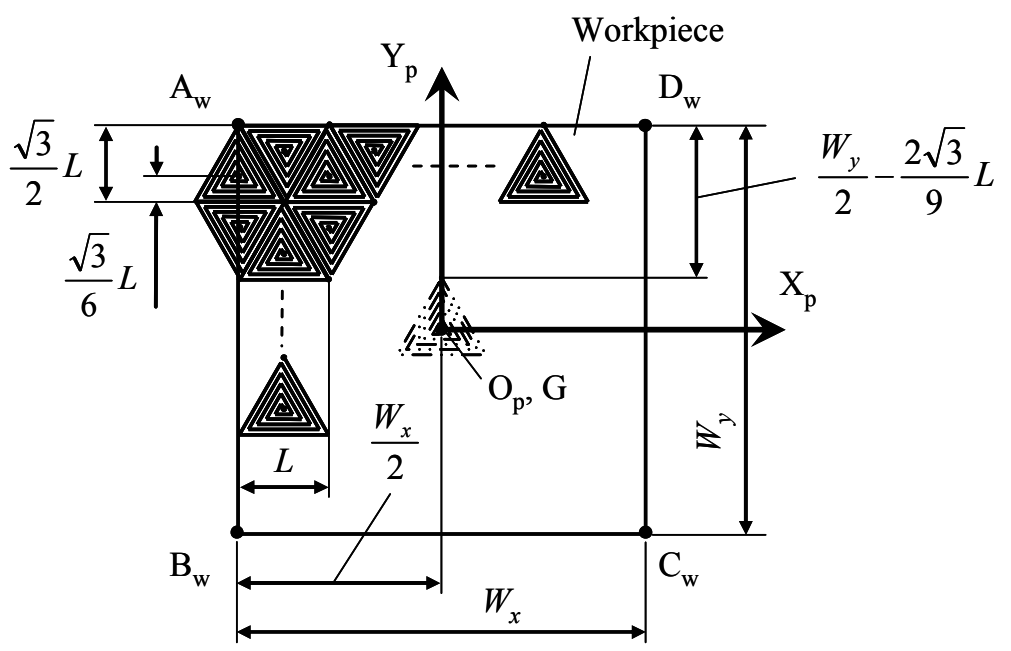

(b) Arrangement of the triangular reference patch on the flat surface

Fig. 2 Tool path generation for an inclined flat surface

2(a). The cross feed $f_{c}$ is kept constant, and $n$ shows the cycle number of the helical tool path. The center of gravity $\mathrm{G}$ of the regular triangle patch is matched to the origin of the $\mathrm{X}_{\mathrm{p}} \mathrm{Y}_{\mathrm{p}}$ plane. This regular triangle patch is called a reference patch. Points $\mathrm{A}_{0}, \mathrm{~B}_{0}$, and $\mathrm{C}_{0}$ are located at $\left(x_{a 0}, y_{a 0}\right),\left(x_{b 0}, y_{b 0}\right)$, and $\left(x_{c 0}, y_{c 0}\right)$, respectively. Point $\mathrm{B}_{0}$ moves along the helical tool path toward the center of gravity $\mathrm{G}$ at every cycle. The transition of the location of Point $B_{n}$ is then given by Eq. (1):

$$
\left[\begin{array}{c}
x_{b_{n}} \\
y_{b_{n}}
\end{array}\right]=\left[\begin{array}{c}
x_{b_{0}}-\frac{n f_{c}}{\tan 60^{\circ}} \\
y_{b_{0}}+n f_{c}
\end{array}\right]
$$

Point $\mathrm{C}_{\mathrm{n}}$ is also given as follows:

$$
\left[\begin{array}{l}
x_{c_{n}} \\
y_{c_{n}}
\end{array}\right]=\left[\begin{array}{c}
x_{c_{n}}+\frac{n f_{c}}{\tan 60^{\circ}} \\
y_{c_{0}}+n f_{c}
\end{array}\right]
$$

Machining starting point $\mathrm{A}_{0}$ moves to point $\mathrm{D}_{\mathrm{n}}$ when the helical tool path goes to the second loop. After that, point $D_{n}$ moves along the $Y_{p}$-axis after the second cycle. The coordinate value of point $D_{n}$ is given as follows:

$$
\left[\begin{array}{l}
x_{d_{n}} \\
y_{d_{n}}
\end{array}\right]=\left[\begin{array}{c}
x_{a_{0}}-f_{c} \tan 30^{\circ} \\
y_{a_{0}}-f_{c}-\frac{n f_{c}}{\sin 30^{\circ}}
\end{array}\right]
$$

The reference patches are then aligned to cover the entire workpiece surface with no space between them. The coordinates of the machining tool path in each patch are transformed into the machine coordinate system of the machine tools used for the machining. The workpiece length in the $\mathrm{X}_{\mathrm{p}}$-axis direction of $W_{x}$ and the length in the $\mathrm{Y}_{\mathrm{p}}$-axis direction of $W_{y}$ 
is determined on the XY plane, and the center of gravity of the workpiece is matched to the center of gravity of the reference patch as shown in Fig. 2(b). The machining starting point $\mathrm{A}_{\mathrm{w}}$ is determined, and thus the starting point on the reference patch is moved to point $\mathrm{A}_{\mathrm{w}}$. However, it is necessary to rotate the center of gravity of the triangular patch alternately by 180 degrees and to correct the gap in the $\mathrm{Y}_{\mathrm{p}}$-axis direction when the regular triangle reference patch is aligned with the $\mathrm{X}_{\mathrm{p}}$-axis direction. When the number of patches in the $\mathrm{X}_{\mathrm{p}}$-axis direction and the $\mathrm{Y}_{\mathrm{p}}$-axis direction are represented by $i$ and $j$, respectively, the transforming matrix $E_{T r}$ used to align the regular triangle reference patch on the workpiece surface is given by Eq. (4):

$$
E_{T_{r}}=\left[\begin{array}{cccc}
\cos (i \pi) & -\sin (i \pi) & 0 & \frac{L(i-\pi)-W_{x}}{2} \\
\sin (i \pi) & \cos (i \pi) & 0 & \frac{W_{y}-\sqrt{3} L(j-1)}{2}+\frac{\sqrt{3}}{6} L \sin ^{2}\left(\frac{i \pi}{2}\right) \\
0 & 0 & 1 & 0 \\
0 & 0 & 0 & 1
\end{array}\right]
$$

Therefore, the NC program, which determines the tool path on the inclined flat surface in patch division milling, can be calculated by multiplying the coordinate transformation matrix $E_{T r}$, the rotation matrix to give the inclination to the $\mathrm{X}_{\mathrm{p}} \mathrm{Y}_{\mathrm{p}}$ plane, and the transformation matrix to transform into the machine coordinate system.

\section{Application of patch division milling to a curved surface}

To apply patch division milling to a curved surface, it is necessary to establish a method for segmenting the machined surface into patches and for generating a tool path. In this chapter, the system for dividing the curved surface of a model designed by 3D-CAD into many triangular patches by means of a FEA preprocessor and the calculation of the helical tool path based on coordinates in the vertices are described.

\subsection{Method of a patch segmentation of a curved surface}

As well as containing a reference patch, the entire face of a finite curved surface should be covered with continuous patches. However, the whole face cannot be covered with only reference patch without a gap when the finite curved surface is divided with the patches. Therefore, when patch division milling is applied to a curved surface, the tool path should be generated after the surface of the workpiece has been divided into patches (with the size of each patch differing according to its location). This can be elucidated by approximating the complex curved surface using a polyhedron mesh well commonly employed in the field of Computer Graphics ${ }^{(14)}$.

In the present study, a finite element analysis preprocessor (FEMAP, UGS Corp.) is used to divide the curved surface into patches. The FEA preprocessor's role is to produce a finite element model by dividing the input area geometrically. This is used to divide the target surface in the workpiece designed by 3D-CAD. In this case, the element model uses a triangular patch. The patch division procedure is performed by a FEA preprocessor, and a side length and an interior angle in each triangular patch are different. All triangular patches that compose the curved surface have an element number. Additionally, all the element numbers have a node number, and the coordinate value corresponding to each node number is preserved in the text file. The element number is preserved in the element file, and the coordinate value of each node number is preserved in the node file. The output from the element file and the node file is used to generate the helical tool's path in processing the inside of each patch based on the node's coordinate value.

\subsection{Tool path generation for a patch arranged on a curved surface}

The element number and the node number composed of the element number are preserved in the element file. Three node numbers are included in one element number because the designed curved surface is divided into triangular patches. Each node number's coordinate value in the direction of the $\mathrm{X}$-axis, the $\mathrm{Y}$-axis, and the $\mathrm{Z}$-axis as defined using 


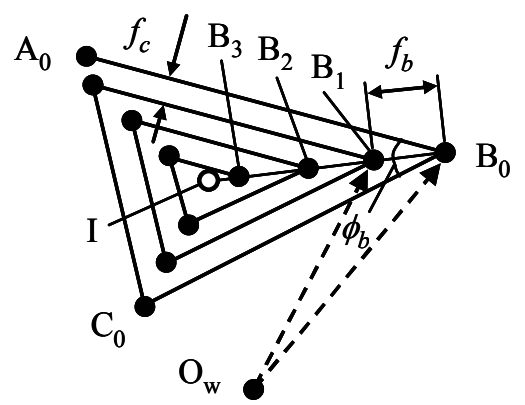

Fig. 3 Helical tool generation in triangular patch

the 3D-CAD system is preserved in the element file. The node number is read from the element file to generate the tool path, and the coordinate value of each node number is preserved in the cache memory. Three node numbers preserved with the element number are then read from the element file, and it is replaced with the coordinate value corresponding to the node number. A helical tool path for a triangular patch is generated from the obtained coordinate value of each node number. In the developed program, the cross feed, the spindle speed, and the feed speed can be input as an initial values. The NC program is calculated by transforming the $3 \mathrm{D}-\mathrm{CAD}$ coordinate system into the machine coordinate system. The method of calculating a helical tool path based on the three vertices of the triangular patch can be described as follows.

As shown in Fig. 3, triangle $\mathrm{A}_{0} \mathrm{~B}_{0} \mathrm{C}_{0}$ is one of the triangular patches into which the curved surface is divided. The origin of the workpiece coordinate system $\mathrm{O}_{\mathrm{w}}$ is the origin of the coordinate system of the $3 \mathrm{D}-\mathrm{CAD}$. Point $\mathrm{B}_{0}$ in the triangular patch shown in Fig. 3 is the focus of this explanation. The method of calculating the position vector in the workpiece coordinate system $\mathrm{O}_{\mathrm{w}}$ when point $\mathrm{B}_{0}$ moves to point $\mathrm{B}_{1}$ is described. Point $\mathrm{B}_{0}$ moves toward incenter $\mathrm{I}$ of the triangular patch $\mathrm{A}_{0} \mathrm{~B}_{0} \mathrm{C}_{0}$ along the helical tool path at every cycle at a constant distance $f_{b}$. Angle $\mathrm{A}_{0} \mathrm{~B}_{0} \mathrm{C}_{0}$ is bisected with vector $\mathrm{B}_{0} \mathrm{~B}_{1}$. Therefore, angle $\mathrm{A}_{0} \mathrm{~B}_{0} \mathrm{C}_{0}$ represented by $\phi_{\mathrm{b}}$ is given by Eq. (5):

$$
\phi_{b}=\cos ^{-1}\left(\frac{\overrightarrow{B_{0} C_{0}} \cdot \overrightarrow{B_{0} A_{0}}}{\left|\overrightarrow{B_{0} C_{0}}\right|\left|\overrightarrow{B_{0} A_{0}}\right|}\right)
$$

The distance $f_{b}$ between point $\mathrm{B}_{0}$ and point $\mathrm{B}_{1}$ is given by Eq. (6):

$$
f_{b}=\frac{f_{c}}{\sin \left(\frac{\phi_{b}}{2}\right)}
$$

Vector $\mathrm{B}_{0} \mathrm{I}$ is given as follows:

$$
\overrightarrow{B_{0} I}=\left(\frac{\left|\overrightarrow{A_{0} B_{0}}\right|}{\left|\overrightarrow{A_{0} B_{0}}\right|+\left|\overrightarrow{B_{0} C_{0}}\right|+\left|\overrightarrow{A_{0} C_{0}}\right|}\right) \times \overrightarrow{B_{0} C_{0}}+\left(\frac{\overrightarrow{B_{0} C_{0}} \mid}{\left|\overrightarrow{A_{0} B_{0}}\right|+\left|\overrightarrow{B_{0} C_{0}}\right|+\left|\overrightarrow{A_{0} C_{0}}\right|}\right) \times \overrightarrow{B_{0} A_{0}}
$$

Vector $\mathrm{B}_{0} \mathrm{~B}_{1}$ is $f_{b}$ times the unit vector in vector $\mathrm{B}_{0} \mathrm{I}$; this is given by Eq. (8):

$$
\overrightarrow{B_{0} B_{1}}=\frac{\overrightarrow{B_{0} I}}{\left|\overrightarrow{B_{0} I}\right|} \times f_{b}=\frac{\overrightarrow{B_{0} I}}{\left|\overrightarrow{B_{0} I}\right|} \times \frac{f_{c}}{\sin \left(\frac{\phi_{b}}{2}\right)}
$$

Therefore, the position vector of point $B_{n}$ in the workpiece coordinate system is given by Eq. (9) when $n$ is the cycle number of the helical tool path.

$$
\overrightarrow{O_{w} B_{n+1}}=\frac{\overrightarrow{B_{n} I}}{\left|\overrightarrow{B_{n} I}\right|} \times \frac{(n+1) f_{c}}{\sin \left(\frac{\phi_{b}}{2}\right)}+\overrightarrow{O_{w} B_{n}}
$$

The position vector of vertex $B_{n}$ at each cycle number is calculated by Eq. (9). The components of the other vertices, vertex $A_{n}$ and vertex $C_{n}$, at each cycle number can be 


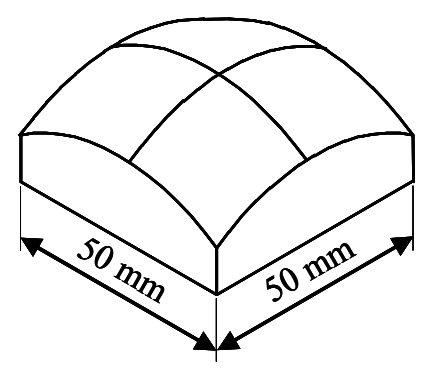

(a) Spherical surface

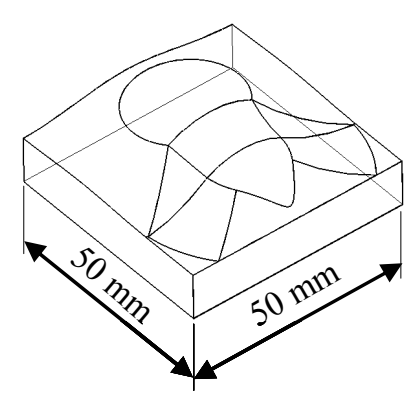

(b) Free-form surface

Fig. 4 Curved surface designed by 3D-CAD

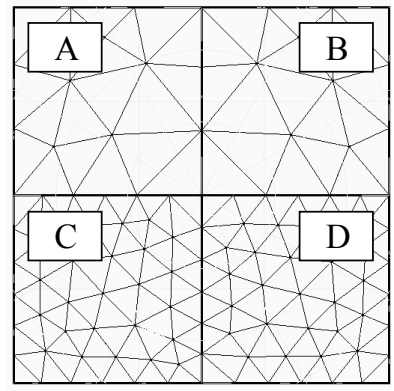

(a) Spherical surface

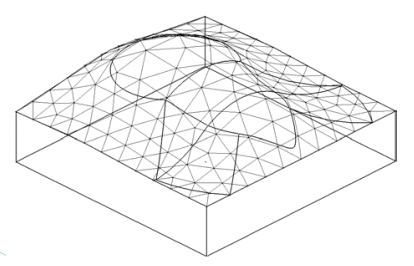

Element number $=247$

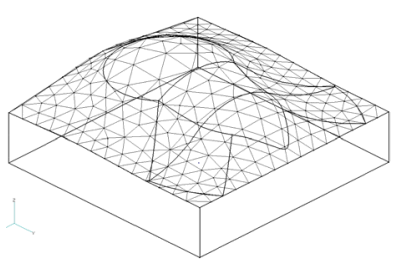

Element number $=413$

Fig. 5 Curved surface divided into triangular patches by FEM preprocessor

estimated in a similar way using Eq. (9).

From the above, it is found that the helical tool path over a triangular patch can be calculated based on the coordinate points at each cycle number in the three vertices. The NC data is then obtained by transforming the coordinate points of the tool path in all triangular patches into the machine coordinate system. Finally, the tool path without tool interference is added between the machining ending point in the patch and the machining starting point in the next patch.

\section{Experimental method and results of the machining of a curved surface}

\subsection{Cutting method and cutting conditions}

A machining experiment was conducted involving a machining center and a solid carbide ball-end mill. The radius of the ball-end mill was $5 \mathrm{~mm}$, and it has 2 flutes. $70 \% \mathrm{Cu}-30 \% \mathrm{Zn}$ brass was used because the favorable machined surface could be easily realized. The pattern of the machined surface was observed with a CCD camera. The angle of the tool during the machining of each patch was not kept constant because only three translation feed axes were used. As shown Fig. 4, a spherical surface (curvature radius: 50 $\mathrm{mm}$ ) and a free-form surface, as designed by 3D-CAD, were machined. The spherical surface, as shown in Fig. 4(a), was divided into four areas. These areas were divided into triangular patches by the FEA preprocessor as shown in Fig. 5(a). As shown in Fig. 5(b), the numbers of patches on the free-form surface were 247 and 413 . The average value of side length in the triangular patch can be input into the FEM preprocessor used in this study. Therefore, as shown in Fig. 5, the patch division with different patch sizes on the same curved surface is possible. The cutting conditions for the spherical surface and the free-form surface are shown in Table 1 and Table 2. In area A and area B of the spherical surface, the number of patches is the same and the patch size between the two areas is symmetrical. Only the number of patches in area $\mathrm{C}$ and area $\mathrm{D}$ are the same. And then, the cross feed of areas $\mathrm{A}$ and $\mathrm{B}$ differ, as do those of areas $\mathrm{C}$ and $\mathrm{D}$.

The reason for dividing the spherical surface and determining the cutting conditions can be described as follows. When an inclined flat surface is machined by patch division milling, the cutter mark array that forms the surface pattern is dominated by the cross feed, the feed 
Table 1 Cutting conditions for spherical surface

\begin{tabular}{|c|c|c|c|c|c|}
\hline & & Area A & Area B & Area C & Area D \\
\hline Spindle speed $S$ & $\min ^{-1}$ & \multicolumn{4}{|c|}{1000} \\
\hline Feed speed $F$ & $\mathrm{~mm} / \mathrm{min}$ & \multicolumn{4}{|c|}{1000} \\
\hline Feed per tooth $f_{t}$ & $\mathrm{~mm} /$ tooth & \multicolumn{4}{|c|}{0.5} \\
\hline Cross feed $f_{c}$ & $\mathrm{~mm}$ & 0.25 & 0.19 & 0.25 & 0.19 \\
\hline Curvature radius & $\mathrm{mm}$ & \multicolumn{4}{|c|}{50} \\
\hline Tool diameter & $\mathrm{mm}$ & \multicolumn{4}{|c|}{5} \\
\hline Number of teeth $N$ & & \multicolumn{4}{|c|}{2} \\
\hline Type of patch & & \multicolumn{4}{|c|}{ Triangle } \\
\hline
\end{tabular}

Table 2 Cutting conditions for free-form surface

\begin{tabular}{|lr|l|}
\hline Spindle speed $S$ & $\mathrm{~min}^{-1}$ & 2000 \\
\hline Feed speed $F$ & $\mathrm{~mm} / \mathrm{min}$ & 1000 \\
\hline Feed per tooth $f_{t}$ & $\mathrm{~mm} /$ tooth & 0.25 \\
\hline Cross feed $f_{c}$ & $\mathrm{~mm}$ & 0.25 \\
\hline Tool diameter & $\mathrm{mm}$ & 5 \\
\hline Number of teeth $N$ & & 2 \\
\hline Type of patch & & Triangle \\
\hline
\end{tabular}
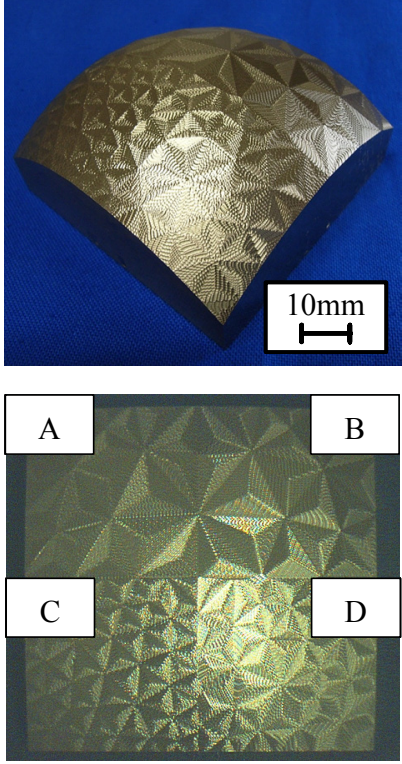

(a) Overall view and top view

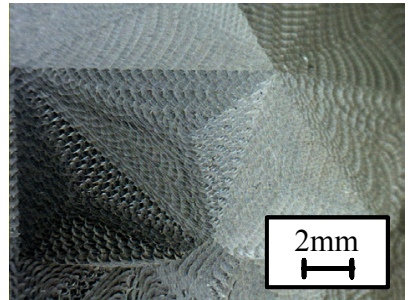

(b) Enlarged view, Area A

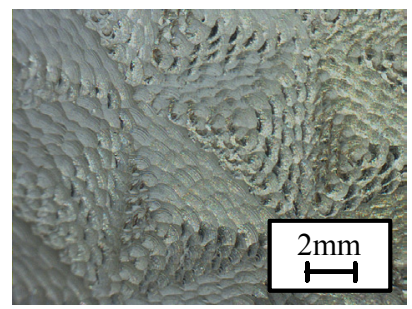

(d) Enlarged view, Area C

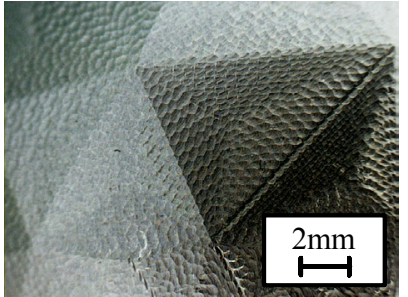

(c) Enlarged view, Area B

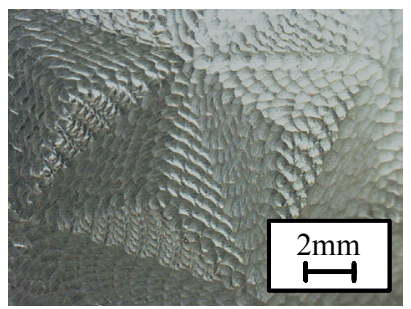

(e) Enlarged view, Area D

Fig. 6 Spherical surface after patch division milling

speed per tooth, the number of teeth, and the patch size (the side length of the patch) and can be controlled by adjusting these parameters ${ }^{(12)(13)}$. Therefore, because a curved surface is approximated by a flat, triangular patch, parameters similar to those involved in machining an inclined flat surface can be adjusted and the difference of the cutter mark array in each patch is checked. Moreover, the difference of the cutter mark array on the free-form surface obtained after changing the patch size is noted.

\subsection{Surface pattern generation on a curved surface by patch division milling}

Figure 6 shows the results of the experiment regarding a spherical surface which has been divided into four areas. As shown Fig. 6(a), it was found that the surface pattern was formed according to the patch's outline on the spherical surface, and that an aligned surface pattern was formed by cutter marks. In addition, it can be seen that the pattern in each patch is divided into a triangular area formed by the sides and the incenter of the patch. A comparison of Fig. 6(b) and Fig. 6(c) shows that the cutter mark array in each patch differs when the number of patches is the same 


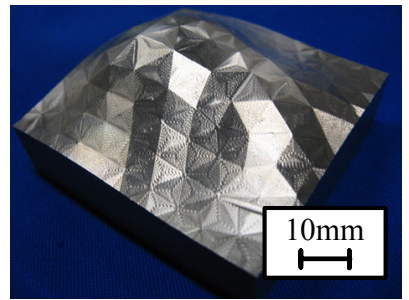

(a) Overall view

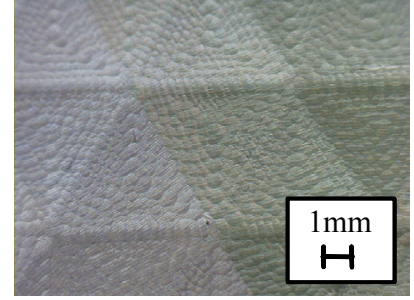

(b) Enlarged view

Figure 7: Free-form surface after patch division milling $($ Element number $=247)$

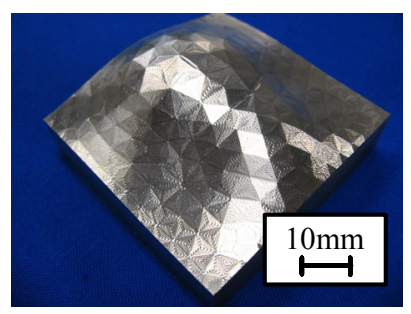

(a) Overall view

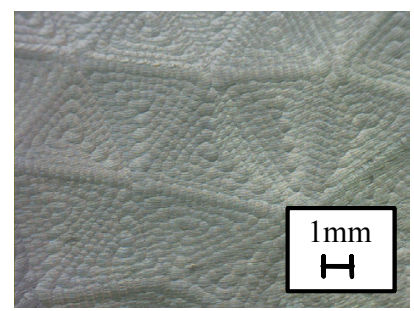

(b) Enlarged view

Figure 8: Free-form surface after patch division milling $($ Element number $=413)$

and the patch size is symmetrical. Therefore, the cutter mark array on the surface altered by the change in the cross feed could be checked. A comparison of the surface patterns of Fig. 6(b) and Fig. 6(d) and of Fig. 6(c) and Fig. 6(e) show that the cutter mark array on the surface differs from the condition in which the cross feed is the same and the patch size is different.

Figure 7 and Figure 8 show results of machining free-form surfaces which have been divided into 247 and 413 triangular patches, respectively. As shown in Fig. 7(a) and Fig. $8(\mathrm{a})$, a surface pattern is generated by the outline of the triangular patch over the entire surface in the same manner as in a spherical surface. Moreover, as shown Fig. 7(b) and Fig. 8 (b), a cutter mark array is generated in each triangular patch. In addition, it is found that the cutter mark array is altered by changing the patch size, as was seen in the experimental results regarding the spherical surface. A difference is seen in the cutter mark array in each patch on a single spherical surface shown in Fig. 6 and on the free-form surfaces shown in Fig. 7and Fig. 8. This is due to the difference in the sizes of the triangular patches on the same curved surface divided by the FEA preprocessor. To resolve these issues and to make the cutter mark array in all patches on a single surface uniform, it is necessary to incorporate an algorithm that adjusts the cutting conditions of each patch into the tool path generation system.

The above demonstrates that patch division milling can be used on various curved surfaces, as based the experimental application of this method to both spherical and free-form surfaces. And then, it is found that patch division milling can be applied to machining on the complex curved surface such as automobile signal lights and reflectors used in lighting equipment.

\section{Conclusion}

In this study, a tool path generation system for patch division milling of a curved surface was described. The system was applied to a curved surface designed by 3D-CAD, and the experiment was conducted. The study's main points can be described as follows:

1) A model of a curved surface designed by $3 \mathrm{D}-\mathrm{CAD}$ was divided into many triangular patches by the FEA preprocessor. A system able to automatically generate a helical tool path in the entire interior area of each patch was developed.

2) A method of calculating the helical tool path based on the coordinates of the three vertices of the triangular patches was demonstrated. 
3) An NC program to machine both a spherical surface and a free-form surface was created using the developed tool path generation system, and the program was tested experimentally. The results showed that patch division milling can be used on free-form surfaces through the use of triangular patch patterns generated over the entire surface of the workpiece to be machined, with this pattern generated by the cutter mark array of each patch.

\section{References}

(1) B, José., T, Uwe., Q, David., Wetting of textured surfaces, Colloids and Surfaces A: Physicochemical and Engineering Aspects, Vol.206, No. 7, (2002), pp.41-46.

(2) Moronuki, N., Takayama, N., and Kaneko, A., Design of surface texture for the control of wettability, Trans. Journal of the Japan Society of Mechanical Engineering, Series B, Vol.70, No. 693, (2004), pp.126-131, (in Japanese).

(3) Z, Liang., F, Yanying., Y, Xiongying., Z, Zhaoying., Tuning wettability and getting superhydrophobic surface by controlling surface roughness with well-designed microstructures, Sensors and Actuators, Vol.130, No. 8, (2006), pp.595-600.

(4) W, Xiaolei., Kato, K., Adachi, K., Aizawa, K., Loads carrying capacity map for the surface texture design of $\mathrm{SiC}$ thrust bearing sliding in water, Tribology International, Vol.36, No. 3, (2003), pp.189-197.

(5) Ito, H., Kaneda, K., Yuhta, T., Nishimura, I., Yasuda, K., Matsuno, T., Reduction of polyethylene wear by concave dimples on the frictional surface in artificial hip joints, The Journal of Arthroplasty, Vol.15, No. 4, (2000), pp.332-338.

(6) Matsuda, H., Sasahara, H., Tsutsumi, M., Influence of Acceleration/Deceleration of Translational Axes and Spindle Speed Deviations on Cutter Mark Array, Journal of the Japan Society for Precision Engineering, Vol.73, No. 3, (2007), pp.355-359, (in Japanese).

(7) Zhao, X., Koreta, N., Tsutsumi, M., Surface roughness generated by ball-end mill on five-axis controlled machining centers. Journal of the Japan Society for Precision Engineering, Vol.64, No. 12, (1998), pp.1826-1830, (in Japanese).

(8) Hao, M., Terai, H., Mizugaki, Y., Theoretical analysis on generating mechanism of machined surface in ball-nosed end milling for spherical surface, Journal of the Japan Society for Precision Engineering, Vol.65, No. 10, (1999), pp.1476-1480, (in Japanese).

(9) C, Jenq-Shyong., H, Yung-Kuo., C, Mao-Son., A study of the surface scallop generating mechanism in the ball-end milling process, International Journal of Machine Tools \& Manufacture, Vol.45, No. 1, (2005), pp.1077-1084.

(10) Saito, A., Zhao, X., Tsutsumi, M., Control of surface texture of mold generated by ball-end milling, Journal of the Japan Society for Precision Engineering, Vol.66, No. 3, (2000), pp.419-423, (in Japanese).

(11) Saito, A., Zhao, X., Tsutsumi, M., A method for generating regular patterns on a curved surface by ball-end milling, Journal of the Japan Society for Precision Engineering, Vol.66, No. 12, (2000), pp.1963-1967, (in Japanese).

(12) Matsuda, H., Sasahara, H., Takizawa, M., Tsutsumi, M., Generation of geometric pattern on plane surface by patch division milling, Journal of the Japan Society for Precision Engineering, Vol.72, No. 9, (2006), pp.1123-1127, (in Japanese).

(13) Matsuda, H., Sasahara, M., Tsutsumi, M., Generation of a regularly aligned surface pattern and control of cutter marks array by patch division milling, International Journal of Machine Tools \& Manufacture, Vol.48, No. 1, (2008), pp.84-94.

(14) Yamamoto, T., Suzuki, H., Kanai, T., Kimura, F., Extended triangular mesh subdivision methods and their evaluation, Journal of the Japan Society for Precision Engineering, Vol.65, No. 3, (1999), pp.386-390, (in Japanese). 